\title{
A Demodulation Algorithm for Time-Phase Modulation Based on Nonstationary Signal Analyzing
}

\author{
Zhongyang Mao \\ Department of Electronic Information Engineering \\ Naval Aeronautical and Astronautical University \\ Yantai 264001, China \\ China Electronic system corp \\ Beijing 100000, China
}

\author{
Hongxing Wang, Xiao Liu, Zhiyong Zhao and \\ Xiguo Liu \\ Department of Electronic Information Engineering \\ Naval Aeronautical and Astronautical University \\ Yantai 264001, China
}

\begin{abstract}
In this paper, the time-frequency distribution property of Time-Phase modulation (TPM) signal is discussed based on nonstationary signal analyzing method. And the mapping relationship between abrupt phase change and time-frequency distribution amplitude in TPM signal is further explored, whereby a TPM signal demodulation algorithm is proposed. Furthermore, this paper gives the performance analysis of the demodulation algorithm and investigates the problem of optimum decision threshold. Simulation is carried out to verify the validity of the theoretical analysis. And the results show that the demodulation algorithm proposed in this paper can offer better error performance than that of traditional correlation demodulation for TPM system.
\end{abstract}

Keywords-bandwidth efficiency; time-phase modulation; abrupt phase change; nonstationary signal; time-frequency distribution

\section{INTRODUCTION}

With the development of the information society, capacity demand of communication systems is getting increasingly greater. Whereas the frequency resource available is rather limited, which tends to exert a bottleneck effect on communication system advancements. Therefore, in order to transmit high rate data within given restricted frequency band, we have to increase bandwidth efficiency to a higher level as possible as we can $[1,2]$. However, the increasing of bandwidth efficiency should not sacrifice too much power efficiency. This suggests that the modulation and demodulation in traditional digital communication theory need to be reconsidered in a new perspective [3-6].

In the traditional digital modulation, the parameters of carrier which can be used to carry digital information are amplitude, phase or frequency. Since the transmitted data is independent of time, the modulated signals could be taken as stationary signals. Nowadays, nonstationary signal processing has been an active area of research, and the relevant processing techniques are becoming more and more mature and some are put into practice [7,8]. In Ref. [9], TPM was proposed based on nonstationary signal analysis, where digital information is transmitted through time-variant signals. The time-variant property of modulation signal means that if the carrier parameter carrying information is amplitude, phase or frequency, the slight distortion after channel transmission would cause the complete loss of information. In TPM modulation, we maps transmitted data into the abrupt phase change at specific moment, ie., the "phase" in TPM is the abrupt phase change rather than the static absolute phase. As a result of abrupt phase change in TPM signal, the traditional correlation demodulation method is no longer a valid alternative now, which leaves the most important problem with us: how to extract the phase jump information in demodulation?

\section{MECHANISM OF TPM}

\section{A. The Signals Model of TPM}

Let us consider binary TPM signal, which can be expressed as:

$$
\begin{gathered}
s(t)=\sum_{n=-\infty}^{+\infty}\left(a_{n} \cdot s_{1}\left(t-n T_{b}\right)+\left(1-a_{n}\right) \cdot s_{0}\left(t-n T_{b}\right)\right) \\
s_{0}(t)=\left\{\begin{array}{l}
A \sin \left(2 \pi f_{c} t+\theta_{1}\right), 0 \leq t \leq \tau_{0} \\
A \sin \left(2 \pi f_{c} t+\theta_{2}\right), \tau_{0}<t \leq T_{b}
\end{array}\right. \\
s_{1}(t)=\left\{\begin{array}{l}
A \sin \left(2 \pi f_{c} t+\theta_{1}\right), 0 \leq t \leq \tau_{1} \\
A \sin \left(2 \pi f_{c} t+\theta_{2}\right), \tau_{1}<t \leq T_{b}
\end{array}\right.
\end{gathered}
$$

Where $f_{c}$ is carrier frequency, $\theta_{2}-\theta_{1}$ is the angle of abrupt phase change, $a_{n}$ is information sequence and $T_{b}$ is bit period.

\section{B. Power Density Spectrum of TPM Signal}

According to Equation(1), the power density spectrum of TPM signal can be derived as:

$$
\begin{gathered}
S_{x x}(f)=\frac{A^{2} \tau^{2}}{4 T \pi^{2}}+\frac{A^{2}(T-\tau)^{2}}{4 T^{2}} f= \pm f_{c} \\
S_{x x}(f)=\frac{A^{2} f_{c}^{2}}{2 T \pi^{2}\left(f_{c}^{2}-f^{2}\right)^{2}}(1-\cos 2 \pi f \tau)+\sum_{\substack{m=-\infty \\
m \neq N}}^{\infty} \delta\left(f-\frac{m}{T}\right) \frac{A^{2} f_{c}^{2}\left(1-\cos \frac{2 \pi \tau m}{T}\right)}{2 T^{2} \pi^{2}\left[f_{c}^{2}-\left(\frac{m}{T}\right)^{2}\right]^{2}} \quad f \neq \pm f_{c}
\end{gathered}
$$

Equation (4) and (5) indicate that the power density spectrum of TPM is made up of continuous spectrum and some linear discrete spectrum. The linear spectrum stands of periodic components of the modulated TPM signal. . 


\section{Demodulation ALGORITHM FOR TIME-PHASE MODULATION BASED ON NONSTATIONARY SIGNAL ANALYZING}

A. The Measure Method of Wave Comparability for TPM Signal Based on Nonstationary Signal Processing

For convenience, and without loss of generality, we assume abrupt phase change of carrier occurs at $t_{0}$, and the phase shift is set to be $\pi$. The received TPM signals are sampled, and a rectangular computing window with width $\mathrm{T}$ is used. Calculating Pseudo Wigner-Wille distribution, when the time instant at which abrupt phase change happens is not contained in the computing window, the time-frequency distribution of TPM signal is

$$
D_{s s}(t, \omega)=\int_{-T / 2}^{T / 2} s(t+\tau / 2) s^{*}(t-\tau / 2) \cdot e^{-j \omega \tau} d \tau=A^{2} \cdot \frac{2 \cdot\left(\sin \left(\omega-\omega_{c}\right) \frac{T}{2}\right)}{\omega-\omega_{c}}
$$

Where $\omega_{c}=2 \pi f_{c}$.

Let $\omega \rightarrow \omega_{c}$, it can be seen that the time-frequency distribution of TPM signal is a constant independent of $t$

$$
\lim _{\omega \rightarrow \omega_{c}} D_{s s}(t, \omega)=A^{2} T
$$

When the phase change occurs within the computing window, the TFD is considered in two different cases. In the First case, we assume the instant lies at the very center of the computing window; in the second case, the instant is assumed to deviate from the center.

Firstly, investigate the first case. Through computation, we have the result of

$$
\begin{gathered}
D_{s s}(t, \omega)=\frac{-A^{2} \cdot\left[\sin \left(\left(\omega-\omega_{c}\right) T\right]\right.}{\omega-\omega_{c}}, \operatorname{T\eta \varepsilon v\Lambda \varepsilon \tau } \omega \rightarrow \omega_{c}, \omega \varepsilon \gamma \varepsilon \tau \\
\lim _{\omega \rightarrow \omega_{c}} D_{s s}(t, \omega)=-A^{2} T
\end{gathered}
$$

Equation (8) shows that the time-frequency distribution at carrier frequency is a positive constant when abrupt phase change does not happen. Whereas it turns negative when the abrupt phase change happens at the center of computing window.

Secondly, let's consider the other case. Assuming that the phase change distant deviates from the center of computing window by $\tau / 2 \quad(\tau>0)$. Wherein the PWVD is given by

$$
D_{s s}(t, \omega)=\frac{4 A^{2} \cdot \sin \left(\left(\omega-\omega_{c}\right) \frac{T}{4}\right.}{\omega-\omega_{c}}-\int_{\tau / 2}^{\tau / 2} 2 \cdot A^{2} \cdot \cos \left[\left(\omega-\omega_{c}\right) \alpha\right] d \alpha
$$

The second term of Equation (9) can be simplified

$$
\begin{aligned}
\int_{\tau / 2}^{\tau / 2} 2 \cdot A^{2} \cdot \cos \left[\left(\omega-\omega_{c}\right) \alpha\right] d \alpha & =\frac{2 A^{2} \cdot\left\{\sin \left[\left(\omega-\omega_{c}\right) \frac{\tau}{2}\right]-\sin \left[\left(\omega-\omega_{c}\right) \frac{T}{2}\right]\right\}}{\omega-\omega_{c}} \\
& =\frac{-4 A^{2} \cdot \cos \left[\left(\omega-\omega_{c}\right) \frac{T+\tau}{4}\right] \sin \left[\left(\omega-\omega_{c}\right) \frac{T+\tau}{4}\right]}{\omega-\omega_{c}}
\end{aligned}
$$

Therefore, the time-frequency distribution in this case is given by

$$
\lim _{\omega \rightarrow \omega_{c}} D_{s s}(t, \omega)=A^{2} \tau-A^{2}(T-\tau)
$$

Equation (11) indicates that the time-frequency distribution at carrier frequency is a line component with energy depending on ${ }^{\tau}$. With the gliding of computing window, at the neighborhood of the point corresponding to the abrupt phase change, the amplitude of the time-frequency distribution decreases continuously towards that point, where the amplitude reaches minimum value. This suggests that if abrupt phase change occurs, there will be a symmetrical reversed triangle gap in time-frequency distribution whose width is equal to the width of computing window. And the valley of the gap corresponds to the instant when the abrupt phase change takes place. Simultaneously, the above analysis provides a measurement method for wave comparison of TPM signals.

\section{B. A TPM Demodulation Algorithm Based on Nonstationary Signal Analyzing}

The results of the above section tell that the time-frequency distribution of TPM signal at carrier frequency is a line component with its energy depending on ${ }^{\tau}$, and there will be a symmetrical reversed triangle gap in time-frequency distribution when phase abrupt change takes place, with its width equal to that of the computing window and the valley corresponding to the instant when the abrupt phase change takes place. Accordingly, a new demodulation algorithm suited to TPM is proposed based on the above discussion. The demodulator structure is illustrated as the figure below

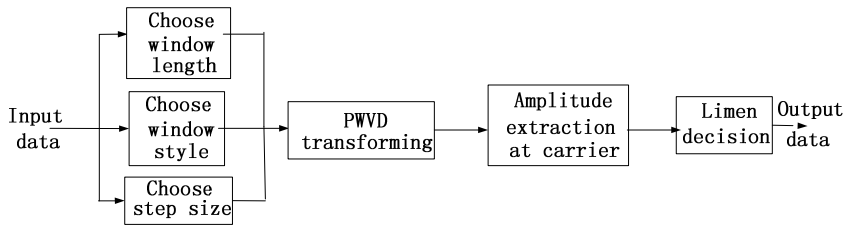

FIGURE I. MODULATOR OF TPM BASED ON NONSTATIONARY SIGNAL ANALYZING

The modulator shown in Figure 1 serves to map abrupt phase change into time-frequency distribution amplitude characteristic. To achieve a better demodulating efficiency, the optimum limen for detection remains to be further discussed.

Steps of the demodulation algorithm are elaborated as follows:

Step 1 Input the modulated signal x(n) into one-rank delay units. The number of delay units depends on the sampling during data period. 
Step 2 Choose the weight coefficient and multiply the data in the delay units by corresponding weight coefficient. The weighting process functions as data window.

Step 3 Calculate the PWVD of windowed data.

Step 4 Extract the amplitude of time-frequency distribution at carrier frequency.

Step 5 Input the amplitude into detector for limen decision, and recover the data information.

\section{PERformance ANAlysis of The TPM DEMOdUlation ALGORITHM}

Assuming additive white Gaussian noise (AWGN) is added to TPM signal, the received signal $r(t)$ at the receiver can be expressed as

$$
r(t)=s(t)+n(t)
$$

Where $s(t), n(t)$ are TPM signal and AWGN respectively.

With discrete data considered, (12) is revised as

$$
r(m)=s(m)+n(m)
$$

Let the width of window be $\mathrm{N}+1$, the time-frequency distribution is given by

$$
P W V D_{r}(m, l)=\sum_{p=-N / 2}^{N / 2} h(p) r(m+p) r(m-p) e^{-j 4 \pi(p l / N)}
$$

Substitute (13) into (14), we have

$$
\begin{aligned}
P W V D_{r}(m, l) & =P W V D_{s s}(m, l)+P W V D_{n n}(m, l) \\
& +P W V D_{s n}(m, l)+P W V D_{n s}(m, l)
\end{aligned}
$$

Where $P W V D_{s s}(m, l)$ and $P W V D_{n n}(m, l)$ are the PWVD of signal and noise respectively, $\quad P W V D_{s n}(m, l)$ and $P W V D_{n s}(m, l)$ are cross PWVD distribution.

For simplicity, assuming ideal symbol synchronous, the decision can be done through sampling the time-frequency amplitude of received signal at the instant when abrupt phase change could take place. Hence, the probability distributions of digital bit " 1 ” and "0" are respectively

$$
\begin{aligned}
& p\left(x_{1}\right)=\frac{1}{\sqrt{2 \pi(N+1) \cdot \sigma_{n}^{2} \cdot\left(2+\sigma_{n}^{2}\right)}} e^{\frac{-\left(x_{1}+(N+1)-\sigma_{n}^{2}\right)^{2}}{2(N+1) \cdot \sigma_{n}^{2} \cdot\left(2+\sigma_{n}^{2}\right)}} \\
& p\left(x_{0}\right)=\frac{1}{\sqrt{2 \pi(N+1) \cdot \sigma_{n}^{2} \cdot\left(2+\sigma_{n}^{2}\right)}} e^{\frac{-\left(x_{0}-(N-1)-\sigma_{n}^{2}\right)^{2}}{2(N+1) \cdot \sigma_{n}^{2} \cdot\left(2+\sigma_{n}^{2}\right)}}
\end{aligned}
$$

The corresponding probability distribution is shown in Figure 2.

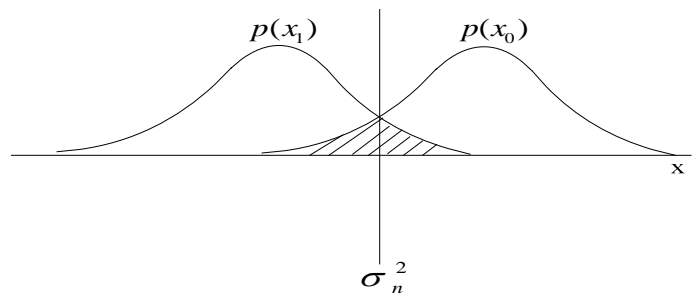

FIGURE II. PROBABILITY DISTRIBUTIONS OF DIGITAL BIT “1”AND " 0 "

Therefore, the optimum decision limen is $\sigma_{n}^{2}$, the probability of error for TPM system can be defined as

$$
P_{e}=(1-P) \cdot p\left(x_{0}<\sigma_{n}^{2}\right)+P \cdot p\left(x_{1}>\sigma_{n}^{2}\right)
$$

Assuming that digital bit “ 1 ” and "0” are equally likely, i.e. $\mathrm{P}=1 / 2$, we obtain

$$
\begin{aligned}
P_{e} & =\frac{1}{2} \int_{-\infty}^{\sigma_{n}^{2}} \frac{1}{\sqrt{2 \pi(N+1) \cdot \sigma_{n}^{2} \cdot\left(2+\sigma_{n}^{2}\right)}} e^{\frac{-\left(x_{0}-(N+1)-\sigma_{n}^{2}\right)^{2}}{2(N+1) \cdot \sigma_{n}^{2} \cdot\left(2+\sigma_{n}^{2}\right)}} d x_{0} \\
& +\frac{1}{2} \int_{\sigma_{n}^{2}}^{\infty} \frac{1}{\sqrt{2 \pi(N+1) \cdot \sigma_{n}^{2} \cdot\left(2+\sigma_{n}^{2}\right)}} e^{\frac{-\left(x_{1}+(N-1)-\sigma_{n}^{2}\right)^{2}}{2(N+1) \cdot \sigma_{n}^{2} \cdot\left(2+\sigma_{n}^{2}\right)}} d x_{1}
\end{aligned}
$$

Finally, the probability of error for TPM system is

$$
P_{e}=Q\left(\frac{\sqrt{N+1}}{\sigma_{n} \sqrt{\left(2+\sigma_{n}^{2}\right)}}\right)
$$

Equation (20) shows that the probability of error for TPM system depends on Signal Noise Ratio (SNR) and width of computing window. Thus, in order to improve the performance of TPM system, higher SNR and broadened data window are two alternatives.. However, increasing SNR means the demand of power, and broadened computing window add to computation complexity. In practice, these two factors are considered according to requirements of power and delay time. In the following simulation, the width of computing is chosen to be two carrier periods.

\section{V.PERFORMANCE SIMULATIONS}

Let carrier frequency $f_{c}=40 \mathrm{kHz}, \tau_{0}=T_{b}, \tau_{1}=1 / 20 T_{b}$, bit rate $f_{b}=2 \mathrm{kHz}$, width of computing window is $2 / f_{c}$, the performance of TPM system with modulator based on nonstationary signal analyzing is shown in Figure 4. The decision limen is adjusted adaptively according to SNR 


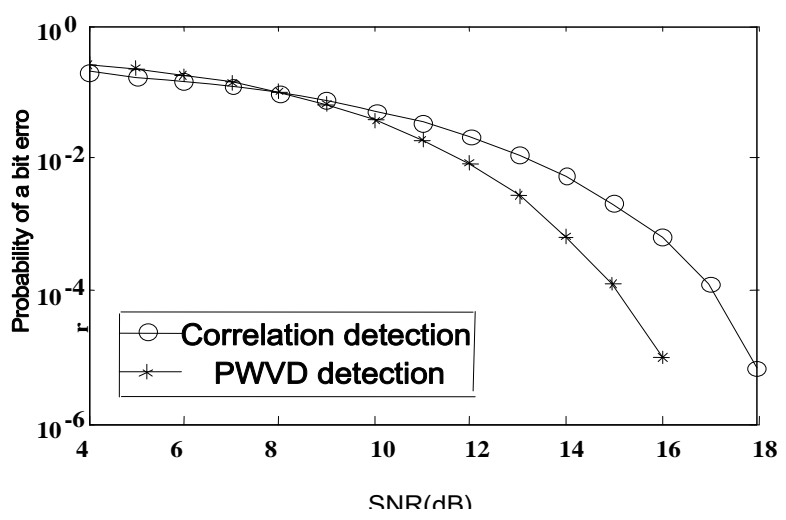

FIGURE III. BER PERFORMANCE OF TPM SYSTEM WITH MODULATOR BASED ON NONSTATIONARY SIGNAL ANALYSING

Figure 3 shows clearly that the time-frequency based demodulation algorithm has a performance better than that of traditional correlation demodulation algorithm.

\section{CONCLUSIONS}

This paper focused on the time-frequency distribution characteristic of TPM modulated signal, and the demodulation algorithm suited to it. By exploring the mapping relationship between the abrupt phase change and the amplitude of timefrequency distribution of TPM signal, a new demodulation algorithm for TPM based on nonstationary signal analysis is presented. The relevant demodulator structure based on the algorithm is proposed. Then the performance evaluation of the demodulation algorithm is conducted, with adaptive decision limen incorporated. The simulation result verified the validity of the demodulation algorithm proposed in the paper which offers a demodulation performance better than that of traditional correlation demodulation.

\section{ACKNOWLEDGEMENT}

This work is supported by National Natural Science Foundation of China (No. 60772056).

\section{REFERENCES}

[1] Liu Cong-Feng. High efficiency Digital Modulation Technique with Application. POSTS \&TELECOM PRESS, Beijing, 2006.

[2] Marvin K. Simon. Bandwidth-Efficient Digital Modulation with Application to Deep Space Communications. TSINGHUA UNIVERSITY PRESS, Beijing, 2006.

[3] H.R. Walker. Textbook . http:// www.vmsk.org. 2009-10-02.

[4] Wu Le-nan. The progress of Ultra Narrow Bandwidth high speed communication. Progress in Natural Science. 2007, 17(11): 1467-1473.

[5] Zhao Cheng-shi. A sine-like wave modulation of xMax. Journal of Beijing University of Posts and Telecommunications, 2007, 30(6): 111115.

[6] Li Bin, Zhou Zheng, Zou Weixia. RPPK Modulation with High Data Rates. Science China Information Sciences, 2010, 53(2): 344-354.

[7] Ge Zhe-que, Chen Zhong-sheng. Matlab time-frequency analysis Technique with Application [M]. POSTS \&TELECOM PRESS, Beijing, 2006

[8] Wang Hong-yu, Qiu Tian-shuang, Chen Zhe. Nonstationary Random Signal Analysis and Processing [M], National Defense Industry Press, Beijing, 2008
[9] Wang Hong-li, Wang Hong-xing, Shu Yi-hao. Time-Phase Modulation Signal Analysis Based on Cyclic Spectrum Correlation Technology [J]. Telecommunication Engineering, Vol. 49, No. 3, pp. 16-20, 2009 